\title{
Use of deep brain stimulation for major affective disorders (Review)
}

\author{
KUANQING MI \\ Department of Neurosurgery, The Fifth People's Hospital of Jinan, Jinan, Shandong 250021, P.R. China
}

Received February 2, 2016; Accepted May 4, 2016

DOI: 10.3892/etm.2016.3622

\begin{abstract}
The multifactorial etiology of major affective disorders, such as major depression and bipolar disorder, poses a challenge for identification of effective treatments. In a substantial number of patients, psychopharmacologic treatment does not lead to effective continuous symptom relief. The use of deep brain stimulation (DBS) for treatment-resistant patients is an investigational approach that has recently produced promising results. The recent development of safer stereotaxic neurosurgery, and the combination with functional neuroimaging to map the affected brain circuits, have led to the investigation of DBS as a potential strategy to treat major mood disorders. Several independent clinical studies have recently shown that chronic DBS treatment leads to remission of symptoms in a high number of treatment-resistant patients for major depression and bipolar disorder. In conclusion, the existing proof-of-principle that DBS can be an effective intervention for treatment-resistant depression opens new avenues for treatment. However, multicenter, randomized and blind trials need to confirm efficacy and be approved after the most recent failures. Patient selection and surgical-related improvements are key issues that remain to be addressed to help deliver more precise and customized treatment.
\end{abstract}

\section{Contents}

1. Introduction

2. Motivation for the use of DBS to treat depression

3. Circuits of depression

4. Results

Correspondence to: Dr Kuanqing Mi, Department of Neurosurgery, The Fifth People's Hospital of Jinan, 24297 Jingshi Road, Jinan, Shandong 250021, P.R. China

E-mail: wilqk2653tux@163.com

Abbreviations: SCC, subcallosal cingulate or Area 25; VC/VS, ventral capsule/ventral striatum; MFB, medial forebrain bundle; HDRS, Hamilton Depression Rating Scale

Key words: depression, deep brain stimulation, bipolar disorder, neurostimulation, treatment-resistant depression
5. Studies in other areas

6. Main sources of variability: Patient selection and surgical imprecision

7. DBS mechanism of action

8. Conclusion

9. The road ahead: The next generation of stimulation systems

\section{Introduction}

Major affective disorders, including depression and bipolar disorder are debilitating neurological diseases that are becoming a burden for society and health systems. Depression or major depressive disorder (MDD) alone affects $6.9 \%$ of the population in Europe (1). It is estimated that at $\geq 20 \%$ of the population suffers from depression during a period of their life (2).

Numerous treatments are currently available, however, these treatments are ineffective, particularly in the field of major depression and in psychiatry in general. Medication and psychotherapy constituted first-line therapies in the treatment of a large spectrum of affective disorders for several decades. For major depression, only a small number of patients respond positively to treatment with drugs and/or psychotherapies, in the form of 'cognitive therapy' (3). In fact, $<40 \%$ of patients achieve remission with first treatment administered.

Converging lines of evidence including neuroimaging, biochemical, electrical signaling, have shown that major depression, as well as other types of affective disorders do not only affect one single region in the brain (4). The multifactorial etiology of major affective disorders, major depression and bipolar disorder, poses a number of challenges for effective treatment. Additionally, due to the absence of reliable biomarkers there is no gold standard to guide an optimal treatment selection for each patient. This is reflected in the trial-and-error combination of different treatments with the aim that the medication or psychotherapy or the combination of the two types may be effective.

A more serious issue is that a majority of patients are unresponsive to multiple medication treatments and psychotherapy, or that medication treatment is unsuccessful over time (5). These severe forms of the disease are referred to as treatment-resistant depression (TRD or refractory depression). For these cases, alternative methods of neurostimulation such as electroconvulsive therapy (ECT), transcranial magnetic 
stimulation (TMS) and vagus nerve stimulation (VNS) have been approved and shown efficacy in the alleviation of certain symptoms (5).

Deep brain stimulation (DBS) is another form of neurostimulation that is an experimental therapy. DBS is an invasive technique that involves the surgical implantation of electrodes bilaterally in the brain, using stereotactic MRI guidance to reach the anatomical target. DBS is an effective and approved treatment for dystonia, hyperkinetic movement disorders such as Parkinson's disease (PD) and several forms of obsessive-compulsive disorder (6). Over the last decade, the safety and therapeutic efficacy of DBS for depression have been successfully tested. DBS has progressively gained ground as a potential strategy to treat TRD (7). Despite the promising first trials, there are surgery-related risks and limitations that remaing to be addressed. Thus, the main aim of this review is to provide an updated overview of the key advances in the use of DBS for the treatment of major mood disorders.

\section{Motivation for the use of DBS to treat depression}

The rationale behind using DBS for the treatment of affective disorders stems from the notion that neuromodulation could serve as a potential strategy. Three key scientific facilitators led to the use of DBS in depression: i) advances in stereotaxic neurosurgery and anatomical precision; ii) experience in other neurological disorders. PD is a neurodegenerative syndrome associated with motor symptoms (tremor, rigidity, bradykinesia) and non-motor deficits (cognitive deficit) that mainly affects thalamic circuits. The pathological hallmark of PD is the progressive loss and death of dopaminergic neurons in the substantia nigra (8). Initially, drugs were used to substitute for the action of dopamine. A better understanding on the basis of the circuit lesion in PD led to the use of high frequency stimulation to act as a substitute (late 80s) for a permanent brain lesion (9). DBS has successfully been used for PD. Specifically, DBS onto the subthalamic nucleus (STN) can totally alleviate tremor, and it is now an FDA-approved treatment (10). Over 100,000 individuals worldwide have this implant. For early and advanced PD, STN DBS appears to be more effective than standard medication $(11,12)$. Notably, after DBS implantation in the globus pallidus internus (GPi) region for PD and tardive dyskinesia, patients have reported significant improvements in mood $(13,14)$. In retrospect, the successful use of DBS for PD shows the clever linkage made between pathology, i.e., chemistry, circuits, lesion; and iii) most recent developments of structural/functional imaging. The wiring diagram and targets for treatment of depression remain to be determined.

However, the multifactorial etiology and variable symptomatology of affective disorders poses great challenges for an effective DBS treatment as in the movement disorders.

\section{Circuits of depression}

Kknowledge concerning brain circuits involved in depression and other psychiatric disorders has greatly evolved. Imaging studies in humans mapped which brain lesions led to depression after stroke. A very critical involvement for the prefrontal cortex was suggested (15). Subsequent studies using MRI measurements and postmortem anatomical studies indicated that the prefrontal cortex, but also abnormalities in the ventromedial frontal cortex and also ventral cingulum (VC) appeared to be present in brains of depressed patients (16). Additionally, studies on animal models of stress suggested the shrinkage of the hippocampus in depression (17). These structural findings were followed by functional studies including PET scanning, and fMRI, which detected patterns of hypoactivity and hyperactivity in different areas of the frontal lobe for unipolar and bipolar depression.

In major depression, primary behavioral problem is associated with an increased negative mood, which affects cognition, circadian rhythms, and movement and patterns of activity. The multi-systemic nature of major affective disorders, major depression and bipolar disorder, are challenging with regard to identification of effective treatment. Therefore, in order to 'tune' a circuit by implanting electrodes in the brain (DBS), a key question is whether there is one node, compartment or associated behavior, that is most critical to this disease.

From the patient reports data, the main hypothesis involved the dysregulation of negative mood as the key clinical feature in depression and possibly all other symptoms (cognitive changes, motor slowing and vegetative symptoms) derived from this dysregulation. Consequently, functional imaging tools allowed the direct mapping of brain regions associated with negative mood. When personal sad memories are retrieved by the patient, hyperactivity of the subcallosal cingulate (SCC or Area 25) and, simultaneously the low activity in the PFC were the main changes observed (18). The most critical component for the regulation of negative mood is the SCC. SCC is a critical brain hub for emotional regulation that constitutes a crossroad of path connections in the cortex (19). Additionally, volume decrease and glia loss have been reported in this region in depressed brains (15). Notably, depression recovery following medication treatment (e.g., SSRI, placebo) or with neurostimulation, rTMS, ECT and VNS, was associated with the downregulation of SCC activity (19-22). As discussed below, other candidate regions have been investigated as therapeutic targets in depression, the main focus for the DBS approach has been given to the SCC, as abnormal patterns of activity in this region have been strongly linked with core clinical features of the disorder.

\section{Results}

Mayberg et al attempted to test the safety and utility of implanting electrodes in a group of ill TRD human patients (23). The 6 patients selected had been disabled by the illness for an average of 6.5 years. Treatments including CBT, and ECT as well as combinations of drugs were unsuccessful. After 6 months of low-frequency chronic stimulation bilaterally onto the SCC, similar to parameters used in PD but in different areas involved in the disease, 4 of the 6 patients responded; and 3 of 6 showed total remission. The treatment response is usually determined by a decrease of $\geq 50 \%$ in the Hamilton Depression Rating Scale (HDRS) score, whereas full remission is achieved when the HDRS score equals 'non-depressed' (24). PET scans in responders confirmed that chronic DBS leads to a decrease of activity of SCC confirming the benefit of directly stimulating the SCC.

The pioneering investigations of Mayberg et al were followed up by another open-label, safety and efficacy 
Table I. Key clinical studies of DBS for depression and bipolar disorder. ${ }^{\text {a }}$

\begin{tabular}{|c|c|c|c|c|c|}
\hline Authors (refs.) & Patient no. & Target area & $\begin{array}{l}\text { Total follow-up } \\
\text { time (months) }\end{array}$ & HDRS (\%) & Remarks \\
\hline Mayberg et al (23) & 6 unipolar & SCC & 6 & 55.0 & \\
\hline Lozano et al (25) & 20 unipolar & $\mathrm{SCC}$ & 12 & 48.2 & \\
\hline Kennedy et al (26) & 20 unipolar & SCC & 72 & 64.3 & $\begin{array}{l}\text { Long-term follow-up of } \\
\text { Lozano et al (25) }\end{array}$ \\
\hline Holtzheimer et al (27) & $\begin{array}{l}11 \text { unipolar } \\
\text { and } 7 \text { bipolar }\end{array}$ & SCC & 24 & 69.0 & $92 \%$ response rate \\
\hline Malone et al (30) & 15 unipolar & $\mathrm{VC} / \mathrm{VS}$ & 48 & 53.3 & \\
\hline Bewernick et al (31) & 11 unipolar & NAc & 48 & 41.7 & \\
\hline Schlaepfer et al (32) & 7 bipolar & MFB & 6 & - & $6 / 7$ patients were responders \\
\hline
\end{tabular}

testing of DBS in SCC white matter with a larger sample of patients (25). Of 20 patients studies, there was a $60 \%$ response rate after 6 months of initiation of the stimulation, and $55 \%$ after 1 year. This response was sustained in a long-term follow up (3-6 years) (26) (Table I). A crucial finding of this second study is the absence of relapse in responsive patients.

A further replication of the first two trials on SCC DBS was performed with a key study in 2012 that extended these results to bipolar disorder (27). Comparable results to first clinical trial were obtained: $42 \%$ response rate was achieved after 6 months and $65 \%$ response after 2 years follow up (Table I). The reasons for the progressive improvement over time are unclear, but the observation that patients who achieve remission did not show spontaneous relapses or resistance to the treatment is promising. Of note, there were no differences between bipolar and unipolar depression patients in terms of the trajectory of response. Episodes of mania or hypermania were not induced by DBS in bipolar patients, a side-effect that had been previously reported (28). These promising results including those for bipolar patients have been further replicated in other centers elsewhere (29). Therefore, using SCC as a target area carries the advantage of treating both unipolar and bipolar disorder.

The BROdmann Area 25 DEep brain Neuromodulation (BROADEN) study was designed as a large multicenter, randomized, blinded clinical study of SCC DBS for TRD patients, sponsored by the St. Jude Medical Institute. However, an open letter reported that the trial was cancelled by the FDA due to poor success offered by the futility analysis (not $>17.2 \%$ probability). Considering the positive results obtained in the previous open-label studies, it is noteworthy that BROADEN was invalidated. Inappropriate patient selection or suboptimal DBS targeting may be factors explaining the failure.

\section{Studies in other areas}

Prompted by the positive results of the stimulation of Area 25, other putative brain regions involved in the circuitry of depression have been under study. Three other major publications in the field have reported the effects of targeting with DBS distinct brain regions (Table I). The ventral capsule/ventral striatum (VC/VS) was stimulated in a group of treatment-resistant patients: a $40 \%$ response rate at 6 months was reported (30). Two other clinical studies investigated two areas involved in reward-processing. An open-label study examined the nucleus accumbens (NAc) (31). After 1 year follow up, $50 \%$ of the patients showed a significant remission. In a third study, the target region was the medial forebrain bundle, which is also connected to the SCC and is involved in reward-processing (32). An acute (within days) and important effect of this treatment was reported with 6 responders of 7 patients (Table I). However, the number of patients and the total period of follow up (6 months) was extremely low and these results should be interpreted cautiously. Thus, the three clinical trials demonstrated that multiple regions may be targeted with successful clinical outcome and therefore suggests the need to understand and define the best target for each patient. It is also unclear whether targeting distinct nodes has an impact on a different subset of symptoms.

The first systematic review on the clinical use of DBS on depression recapitulated the rapid progress made in this field over the last decade (33). In total, 22 clinical trials including 188 patients that received DBS for a refractory mood disorder have been published (33). The strongest evidence is for the antidepressant effect of SCC DBS for TRD patients. (69.6\% of a total of 131 patients). At the one-year mark after the onset of treatment, comparable outcomes were independently reported across different groups. Nevertheless, the number of patients in each clinical trial has been low and the inclusion criteria and score test have been overly heterogeneous.

\section{Main sources of variability: Patient selection and surgical imprecision}

From the early use of DBS in depression, PET imaging showed that Area 25 in responders was altered and remote changes in blood flow were present (23). Notably, the brain scans of non-responders indicated that there is a correlation between 
the absence of DBS stimulation reaching these outer areas and poor clinical outcome. Thus, the treatment constitutes not only a local effect, but rather a network effect. The rest of this circuit plays an important role in anti-depressant actions, because the stimulation and downregulation of Area 25 alone is insufficient.

The development of new tools in the realm of white matter tractography such as diffusion tensor imaging (DTI) has made possible examination in great detail of the difference between stimulating one active contact or another (34). A minor change in anatomy can have a major effect in connection. Targeting optimal pathways is relevant to electrode placement and programming. Individual maps of white matter tracts can now be used to identify specific patterns of altered connectivity, which may ultimately define the optimal placement of electrodes. The use of state-of-the-art microstructural scanning opens new avenues for customized DBS treatment. A more refined DBS targeting can prove particularly useful for those cases where multiple brain circuits appear to be affected (35). In this context, the implantation of multipolar-lead electrodes (36), should be carefully examined in future clinical studies.

SCC is a hub for three sets of tracts: midline thalamus, ventral pallidum, and medial frontal cortex (35). The recent modelling of patient-specifc tractography-activation data has shown that the combined stimulation of the three tracts is necessary to achieve full clinical response (37). As larger sets of imaging data from patients are compiled, better correlations between the clinical outcome (responders/non-responders) and the exact targeted pathways can be established, and this may be used to define reliable biomarkers and refine surgical approach. Collecting data on how distinct behavior is impacted by network dysfunction may be useful in mapping specific behaviors to individual brain regions and individual circuits.

A primary assessment of the stimulation efficiency can already occur in the operating room. Although it seems that the response to DBS requires time to come into effect, acute behavioral changes often occur when the correct target is achieved. Patients have shown a number of spontaneous selfreports that predict a positive response (38). A collaborative effort between neurosurgeons and psychiatrists during surgery now allows to test causal relationships in real-time. Monitoring simultaneously physiology, i.e., local field potentials, whole brain activity, SCC activity, and behavioral changes while placing the electrodes provides critical information as to how the patient responds as stimulation is applied second to second.

Stereotactic procedures are highly invasive and $2 \%$ of total DBS implants lead to intracranial bleeding (4). Other serious adverse effects in the long-term follow up of patients have been reported (38), but there is no direct evidence that these were caused by DBS surgery or stimulation programs. In principle, DBS is a reversible procedure, thus stimulation can be switched off or even completely removed if necessary. More detailed and frequent checkup of patients using state-of-the art neuroimaging techniques needs to be implemented to successfully reduce the adverse effects of DBS.

\section{DBS mechanism of action}

The short- and long-term structural and functional changes that occur in the brain in response to DBS stimulation remain to be elucidated. A better understanding of the neurophysiological adaptations triggered by DBS are fundamental to optimize the stimulation parameters, and therefore minimize the adverse side-effects and brain damage.

Microelectrode unit recording during implantation has provided some insight into the information processing in the SCC (39). Electrophysiological recordings from a set of neurons in the SCC as the awake patient in the operating room passively processes images with different emotional valence, shows that Area 25 mostly monitors negative emotions (40). Individual neurons appear to be emotion-specific. Based on the effect of DBS on reducing SCC, a hypothesis that has been posited is that it suppresses negative mood. This raises the question of whether DBS indirect symptomatology affects mood or motivation, as well as some of the vegetative features or cognition.

The identification of the most critical nodes, brain regions in the circuit of depression (36), provides a great opportunity to reverse-engineer this investigation into animal models, and examine the fundamental mechanisms at many levels, i.e., regions, networks, and cell types. In this regard, the use of in vivo optogenetics may be a powerful tool to tease specific networks in animal models of depression and help translate this work for clinical studies.

Another critical issue is whether recovery persists stably after DBS stimulation stops. A possibility is that DBS action catalyzes or induces endogenous plasticity, in order that when the external battery dies, normal brain mechanisms can take over. This hypothesis was tested, and a blinded discontinuation of the stimulation, by simply turning battery on and off, in 3 patients (all received sham stimulation) for a period of 3 months (27). The patients exhibited rapid deterioration and showed relapse after a period of 2 weeks. This was also confirmed by cases of battery depletion. Battery replacement and restart of the active stimulation rescued the effect (27). It remains to be elucidated whether these patients may be rescued with conventional treatments such as medication or therapy.

Higher stimulation voltages showed a correlation with a better clinical outcome (26), and are associated with higher battery use, leading to more frequent replacement of batteries and possible surgical complications. The use of rechargeable batteries (41) and the establishment of pacemaker-like cycles of stimulation, such as successful implants in the treatment of movement disorders $(42,43)$, have been proposed as a solution to this problem. This therapy should take advantage of the developments in the closed-loop stimulation systems (36) to provide discontinuous stimulation, based on the clinical demands of each patient.

\section{Conclusion}

Almost 10 years after the first clinical trial of DBS for depression, progress has been achieved, although further investigations are required to confirm DBS as an extended treatment for major mood disorders. As a therapy, DBS stands in the interface between neurosurgery and interventional psychiatry (44). Multicenter randomized and blind trials need to show efficacy and be approved following recent failures. In addition, which patients are likely to benefit from the treatment remains to be determined. For this purpose, a detailed registry of clinical data from all the patients (including imaging information) and 
open-access data are required to elaborate improved criteria for DBS patient selection. Successful targeting of 6 brain sites for depression has been reported, but little is known regarding the biomarkers that predict better outcomes for each patient.

\section{The road ahead: The next generation of stimulation systems}

The positive clinical outcome of DBS treatment for several neurological disorders in the last few years has sparked a great deal of technological innovation in order to optimize the surgical approach and tailor treatments to each patient. The ambitious Connectome Project aims to provide access to white matter projection maps in an unprecedented resolution (45). In combination with the in vivo DTI tract tracing (46) the connectome dataset aimed to provide precise information concerning the best target networks for each patient. The progress achieved on the imaging branch was matched by the development of more precise neuroengineering devices for DBS surgery. A major concern has been the need to specifically stimulate certain contacts, and avoid a cloud of activity in off-target circuits. The newest microelectrodes enable to actually drive them with high precision (47), as well as targeting multiple leads (36). In future, robotic systems are being optimized for MRI-guided stereotactic surgery (48).

The time course of DBS-induced effects appears to be relatively stereotypic with a few exceptions. Although there is not an immediate or rapid action, certain acute behavioral switches can be predicted in the operating room. At present, there are not reliable and obvious clinical predictors for determining the time course in each patient. Successful DBS treatment requires to be followed by an appropriate post-therapy. Once chronically ill, the now recovering patient must cope with the change of an entire lifestyle, and developing new habits to regain integration within society or family. As recovery takes much more than a stimulator, improvements on the surgical approach must be logically accompanied by the development of effective post-treatment rehabilitation strategies (49). The exact contribution of DBS treatment to the enhancement of brain plasticity and learning may be an important aspect to determine.

Despite being an experimental treatment, the use of DBS for treating affective disorders in humans has already raised evident ethical concerns due to its potential misuse (50). The raise of potential ethical alarms has been partly caused by the comparison with psychosurgery methods used previously (4). Elevated economic costs, as well as safety and tolerability issues hinder the possibility that, in the next 5 years, DBS is likely to gain the status of recommended third-line treatment in most health systems guidelines. Leading figures in the field have elaborated some guidelines for the clinical use of DBS to treat MDD (51). Ultimately, DBS may become a first-line or second-line treatment for refractory depression and bipolar disorder.

\section{References}

1. Wittchen HU, Jacobi F, Rehm J, Gustavsson A, Svensson M, Jönsson B, Olesen J, Allgulander C, Alonso J, Faravelli C, et al: The size and burden of mental disorders and other disorders of the brain in Europe 2010. Eur Neuropsychopharmacol 21: 655-679, 2011.
2. Hasler G: Pathophysiology of depression: do we have any solid evidence of interest to clinicians? World Psychiatry 9: 155-161, 2010.

3. Khan A, Faucett J, Lichtenberg P, Kirsch I and Brown WA: A systematic review of comparative efficacy of treatments and controls for depression. PLoS One 7: e41778, 2012.

4. Kringelbach ML, Jenkinson N, Owen SL and Aziz TZ: Translational principles of deep brain stimulation. Nat Rev Neurosci 8: 623-635, 2007.

5. Rizvi SJ, Donovan M, Giacobbe P, Placenza F, Rotzinger S and Kennedy SH: Neurostimulation therapies for treatment resistant depression: a focus on vagus nerve stimulation and deep brain stimulation. Int Rev Psychiatry 23: 424-436, 2011.

6. Miocinovic S, Somayajula S, Chitnis S and Vitek JL: History, applications, and mechanisms of deep brain stimulation. JAMA Neurol 70: 163-171, 2013.

7. Accolla EA, Aust S, Merkl A, Schneider GH, Kuhn AA, Bajbouj M and Draganski B: Deep brain stimulation of the posterior gyrus rectus region for treatment resistant depression. J Affect Disord 194: 33-37, 2016.

8. Hwang DY, Ardayfio P, Kang UJ, Semina EV and Kim KS: Selective loss of dopaminergic neurons in the substantia nigra of Pitx3-deficient aphakia mice. Brain Res Mol Brain Res 114: 123-131, 2003.

9. Bronstein JM, Tagliati M, Alterman RL, Lozano AM, Volkmann J, Stefani A, Horak FB, Okun MS, Foote KD, Krack P, et al: Deep brain stimulation for Parkinson disease: an expert consensus and review of key issues. Arch Neurol 68: 165-165, 2011.

10. Berney A, Vingerhoets F, Perrin A, Guex P, Villemure JG, Burkhard PR, Benkelfat C and Ghika J: Effect on mood of subthalamic DBS for Parkinson's disease: a consecutive series of 24 patients. Neurology 59: 1427-1429, 2002.

11. Williams A, Gill S, Varma T, Jenkinson C, Quinn N, Mitchell R, Scott R, Ives N, Rick C, Daniels J, et al; PD SURG Collaborative Group: Deep brain stimulation plus best medical therapy versus best medical therapy alone for advanced Parkinson's disease (PD SURG trial): a randomised, open-label trial. Lancet Neurol 9: 581-591, 2010.

12. Schuepbach WM, Rau J, Knudsen K, Volkmann J, Krack P, Timmermann L, Hälbig TD, Hesekamp H, Navarro SM, Meier N, et al; EARLYSTIM Study Group: Neurostimulation for Parkinson's disease with early motor complications. N Engl J Med 368: 610-622, 2013.

13. Kosel M, Sturm V, Frick C, Lenartz D, Zeidler G, Brodesser D and Schlaepfer TE: Mood improvement after deep brain stimulation of the internal globus pallidus for tardive dyskinesia in a patient suffering from major depression. J Psychiatr Res 41: 801-803, 2007.

14. Damier P, Thobois S, Witjas T, Cuny E, Derost P, Raoul S, Mertens P, Peragut JC, Lemaire JJ, Burbaud P, et al; French Stimulation for Tardive Dyskinesia (STARDYS) Study Group: Bilateral deep brain stimulation of the globus pallidus to treat tardive dyskinesia. Arch Gen Psychiatry 64: 170-176, 2007.

15. Drevets WC, Ongür D and Price JL: Neuroimaging abnormalities in the subgenual prefrontal cortex: implications for the pathophysiology of familial mood disorders. Mol Psychiatry 3: 220-226, 190-191, 1998

16. Robinson RG, Kubos KL, Starr LB, Rao K and Price TR: Mood changes in stroke patients: relationship to lesion location. Compr Psychiatry 24: 555-566, 1983.

17. Sapolsky RM: Depression, antidepressants, and the shrinking hippocampus. Proc Natl Acad Sci USA 98: 12320-12322, 2001.

18. Price JL and Drevets WC: Neurocircuitry of mood disorders. Neuropsychopharmacology 35: 192-216, 2010.

19. Hamani C, Mayberg H, Stone S, Laxton A, Haber S and Lozano AM: The subcallosal cingulate gyrus in the context of major depression. Biol Psychiatry 69: 301-308, 2011.

20. George MS, Wassermann EM, Williams WA, Callahan A, Ketter TA, Basser P, Hallett M and Post RM: Daily repetitive transcranial magnetic stimulation (rTMS) improves mood in depression. Neuroreport 6: 1853-1856, 1995.

21. Nobler MS and Sackeim HA: Neurobiological correlates of the cognitive side effects of electroconvulsive therapy. J ECT 24: 40-45, 2008.

22. Pardo JV, Sheikh SA, Schwindt GC, Lee JT, Kuskowski MA, Surerus C, Lewis SM, Abuzzahab FS, Adson DE and Rittberg BR: Chronic vagus nerve stimulation for treatment-resistant depression decreases resting ventromedial prefrontal glucose metabolism. Neuroimage 42: 879-889, 2008. 
23. Mayberg, H, Lozano A, Voon V, McNeely H, Seminowicz D, Hamani C, Schwalb J and Kennedy S: Deep brain stimulation for treatment-resistant depression. Neuron 45: 651-660, 2005.

24. Endicott J, Cohen J, Nee J, Fleiss J and Sarantakos S: Hamilton depression rating scale. Extracted from regular and change versions of the schedule for affective disorders and schizophrenia. Arch Gen Psychiatry 38: 98-103, 1981.

25. Lozano AM, Mayberg HS, Giacobbe P, Hamani C, Craddock RC and Kennedy SH: Subcallosal cingulate gyrus deep brain stimulation for treatment-resistant depression. Biol Psychiatry 64: 461-467, 2008.

26. Kennedy SH, Giacobbe P, Rizvi SJ, Placenza FM, Nishikawa Y, Mayberg HS and Lozano AM: Deep brain stimulation for treatment-resistant depression: follow-up after 3 to 6 years. Am J Psychiatry 168: 502-510, 2011

27. Holtzheimer PE, Kelley ME, Gross RE, Filkowski MM, Garlow SJ, Barrocas A, Wint D, Craighead MC, Kozarsky J, Chismar R, et al: Subcallosal cingulate deep brain stimulation for treatment-resistant unipolar and bipolar depression. Arch Gen Psychiatry 69: 150-158, 2012.

28. Haq IU, Foote KD, Goodman WK, Ricciuti N, Ward H, Sudhyadhom A, Jacobson CE, Siddiqui MS and Okun MS: A case of mania following deep brain stimulation for obsessive compulsive disorder. Stereotact Funct Neurosurg 88: 322-328, 2010.

29. Puigdemont D, Pérez-Egea R, Portella MJ, Molet J, de Diego-Adeliño J, Gironell A, Radua J, Gómez-Anson B, Rodríguez R, Serra M, et al: Deep brain stimulation of the subcallosal cingulate gyrus: furtherevidencein treatment-resistant major depression. Int J Neuropsychopharmacol 15: 121-133, 2012.

30. Malone DA Jr, Dougherty DD, Rezai AR, Carpenter LL, Friehs GM, Eskandar EN, Rauch SL, Rasmussen SA, Machado AG, Kubu CS, et al: Deep brain stimulation of the ventral capsule/ventral striatum for treatment-resistant depression. Biol Psychiatry 65: 267-275, 2009.

31. Bewernick BH, Hurlemann R, Matusch A, Kayser S, Grubert C, Hadrysiewicz B, Axmacher N, Lemke M, Cooper-Mahkorn D, Cohen MX, et al: Nucleus accumbens deep brain stimulation decreases ratings of depression and anxiety in treatment-resistant depression. Biol Psychiatry 67: 110-116, 2010.

32. Schlaepfer TE, Bewernick BH, Kayser S, Mädler B and Coenen VA: Rapid effects of deep brain stimulation for treatment-resistant major depression. Biol Psychiatry 73 : 1204-1212, 2013

33. Morishita T, Fayad SM, Higuchi MA, Nestor KA and Foote KD: Deep brain stimulation for treatment-resistant depression: Systematic review of clinical outcomes. Neurotherapeutics 11 : 475-484, 2014

34. Coenen VA, Kieselbach K, Mader I and Reinacher PC: Diffusion tensor magnetic resonance imaging (DTI) tractography-guided deep brain stimulation in neuropathic pain. Acta Neurochir (Wien) 157: 739-741, 2015.

35. Underwood E: Short-circuiting depression. Science 342: 548-551, 2013.
36. Hariz M: Deep brain stimulation: new techniques. Parkinsonism Relat Disord 20 (Suppl 1): S192-S196, 2014.

37. Lujan JL, Chaturvedi A, Choi KS, Holtzheimer PE, Gross RE, Mayberg HS and McIntyre CC: Tractography-activation models applied to subcallosal cingulate deep brain stimulation. Brain Stimulat 6: 737-739, 2013.

38. Delaloye S and Holtzheimer PE: Deep brain stimulation in the treatment of depression. Dialogues Clin Neurosci 16: 83-91, 2014

39. Abosch A, Lanctin D, Onaran I, Eberly L, Spaniol M and Ince NF: Long-term recordings of local field potentials from implanted deep brain stimulation electrodes. Neurosurgery 71 : 804-814, 2012.

40. Laxton AW, Neimat JS, Davis KD, Womelsdorf T, Hutchison WD, Dostrovsky JO, Hamani C, Mayberg HS and Lozano AM: Neuronal coding of implicit emotion categories in the subcallosal cortex in patients with depression. Biol Psychiatry 74: 714-719, 2013.

41. Malone DA Jr: Use of deep brain stimulation in treatment-resistant depression. Cleve Clin J Med 77 (Suppl 3): S77-S80, 2010.

42. Santaniello S, Fiengo G, Glielmo L and Grill W: Closed-loop control of deep brain stimulation: a simulation study. IEEE Trans Neural Syst Rehabil Eng 19: 15-24, 2011.

43. Kuncel AM, Birdno MJ, Swan BD and Grill WM: Tremor reduction and modeled neural activity during cycling thalamic deep brain stimulation. Clin Neurophysiol 123: 1044-1052, 2012.

44. Williams NR and Okun MS: Deep brain stimulation (DBS) at the interface of neurology and psychiatry. J Clin Invest 123: 4546-4556, 2013.

45. Van Essen DC, Ugurbil K, Auerbach E, Barch D, Behrens TE, Bucholz R, Chang A, Chen L, Corbetta M, Curtiss SW, et al; WU-Minn HCP Consortium: The Human Connectome Project: a data acquisition perspective. Neuroimage 62: 2222-2231, 2012.

46. Basser PJ, Pajevic S, Pierpaoli C, Duda J and Aldroubi A: In vivo fiber tractography using DT-MRI data. Magn Reson Med 44: 625-632, 2000

47. Contarino MF, Bour LJ, Verhagen R, Lourens MA, de Bie RM, van den Munckhof $P$ and Schuurman PR: Directional steering: a novel approach to deep brain stimulation. Neurology 83: 1163-1169, 2014.

48. Li G, Su L, Cole G, Shang W, Harrington K, Camilo A, Pilitsis J and Fischer G: Robotic System for MRI-Guided Stereotactic Neurosurgery. IEEE Trans Biomed Eng 62: 1077-1088, 2015.

49. Riva-Posse P, Holtzheimer P, Garlow S and Mayberg S: Practical considerations in the development and refinement of subcallosal cingulate white matter deep brain stimulation for treatment-resistant depression. World Neurosurg 80: S27.e25-34, 2013.

50. Cleary DR, Ozpinar A, Raslan AM and Ko AL: Deep brain stimulation for psychiatric disorders: where we are now. Neurosurg Focus 38: E2, 2015.

51. Schlaepfer TE, George MS and Mayberg H; WFSBP Task Force on Brain Stimulation: WFSBP Guidelines on brain stimulation treatments in psychiatry. World J Biol Psychiatry 11: 2-18, 2010. 